Planetary Systems in the Universe - Observation, Formation and Evolution

Proceedings IAU Symposium No. 202, (C)2004 IAU

Alan Penny, Pawel Artymowicz, Anne-Marie Lagrange, \& Sara Russell, eds.

\title{
Extra-solar planets: from direct rotation into reverse rotation
}

\section{Irina Kitiashvili}

Kazan University, Dpt. Radioelectronics, Kremljevskaja Str., 18, Kazan, 420008, Russia E-mail: Irina.Kitiashvili@ksu.ru

\author{
Alexander Gusev \\ Kazan University, Dpt. of Gravitation, Kremljevskaja Str., 18, Kazan, \\ 420008, Russia E-mail: Alexander.Gusev@ksu.ru
}

\begin{abstract}
We investigate the equation describing the evolution of the kinetic momentum vector for the case of non-resonance rotation of dynamically symmetrical planets by action of gravitational and magnetic interaction with the central star. The obtained gallery of more twenty phase portraits of kinetic momentum evolution illustrates the various regimes of the planetary systems evolution. The analyses of obtained portraits has shown that a direct rotation of the planet may be passed into reverse rotation and vice versa for a rather broad range of the parameters.
\end{abstract}

The process of planetary formation is significantly determined by the orbitalrotational characteristics of the planet. The rotation of the extra-solar planet is characterized by the position of the kinetic momentum vector in the space and is determined by gravitational and magnetic interrelation with the star. At the present time 47 planetary system are discussed. The search for the planets is carried out by four methods: radio-astronomical, astrometrical, spectroscopic and HST photometric methods (Mayor \& Queloz 1995; Gonzalez 1998; Wolszczan \& Frail 1992). Measurement of Doppler shift of star spectral lines, caused by the ray velocity variation, is in most common use to search for the planets around the stars of main sequence. More than 30 extra-solar planets were discovered by this method, for instance, b Vir around the 36 Uma (Nelson \& Angel 1998).

Most of proposed planets moves around the stars of main sequence with a spectral class F5 - K5 (Fig.1) and are located at distance of 3 pc (epsilon Eridani ) - $3.8 \mathrm{kpc}$ (PSR B1620-26). Among the all extra-solar planets the following classes may be picked out: in accordance with mass - 48 planets with Jupiter's mass;
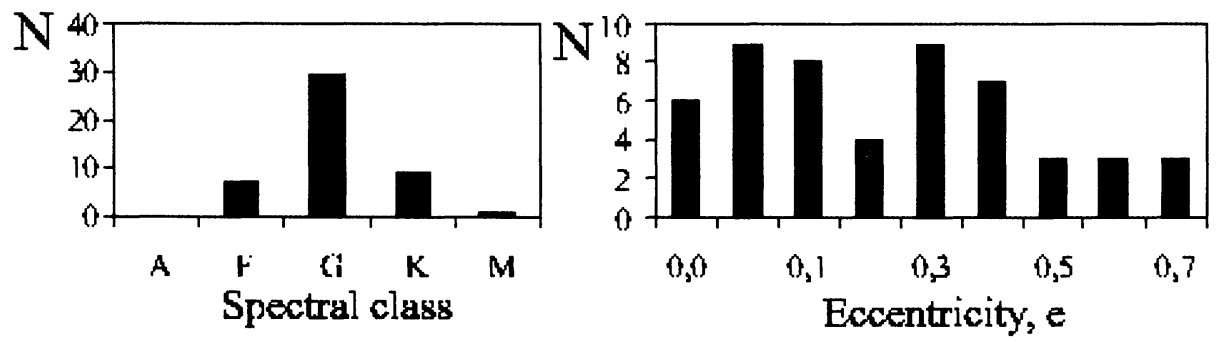

Figure 1. Spectral class of stars and the eccentricity of exoplanets. 
3 - with Earth's mass; in accordance with periods - 33 short-periodic (before 180 days) and 6 long-periodic (more 1000 days) planets. From the catalogue of candidates of extra-solar planets (http://www.obspm.fr/encycl/catalog.html) it is seen: three exoplanets systems contain more than one planet (PSR $1257+12$ - 3 planets, $\nu$ Andromedae - 3 planets, HD 83443 - 2 planets ); practically all planetary systems are analogous to Solar system, the planetary masses therewith lie in the range $0.015 \mathrm{M}_{\oplus}$ and $11 \mathrm{M}_{J}$, the main semi-axes lie between 0.038 a.u. and 2.5 a.u., and the revolution periods are varied from 1.79 to 1619 days; the planets around the pulsars have the mass of order of the Earth's mass, and the planets around the stars of main sequence have the Jupiter's mass $\left(3-7 \mathrm{M}_{J}\right)$.

Structure of planetary systems depends essentially on the effects of their own rotation. Main characteristics of the rotation were obtained by Beletsky and Khentov (1995): they have derived the generalised Kassini's lows for natural celestial bodies and have investigated the mechanism of capture into resonance rotation.

It is known a planetary rotation is determined by orientation of a vector of kinetic moment of a body $\mathrm{L}$ in a space. The Euler angles are convenient to describe the position of the kinetic moment vector. The position of kinetic moment vector is defined relative to a pericenter $\mathrm{P}$ of an osculating orbit, $\omega_{p}$ is the angle equal to the longitude of pericenter of the orbit.

The planet will be considered as a dynamically symmetrical celestial body magnetised along the symmetry axis, its rotation is nonresonant. According to (Beletsky \& Khentov 1995; Kitiashvili 1999), under the total perturbation from gravitational and magnetic moment the following equations describe the evolution of the vector $\mathrm{L}$ :

$$
\begin{aligned}
& \dot{x}=-\alpha \sin y \\
& \dot{y}=\beta-\alpha c t g x \cos y+\gamma \cos x .
\end{aligned}
$$

where $\mathrm{x}, \mathrm{y}$ is Euler angles, $\alpha, \beta, \gamma$ are parameters of magnetic and gravitational moments.

Table 1. Equilibrium states $\beta \neq 0$

\begin{tabular}{|l|c|c|c|}
\hline Coordinates & $\alpha<\gamma$ & $\alpha=\gamma$ & $\alpha>\gamma$ \\
\hline$y=o, x=\frac{\pi}{2}+\frac{\beta}{\gamma-\alpha}$ & saddle & - & center \\
\hline$y=o, x=-\frac{\pi}{2}-\frac{\beta}{\gamma+\alpha}$ & center & center & center \\
\hline$y=o, x=\arcsin \frac{\alpha}{\gamma}-\frac{\beta \alpha}{\beta \sqrt{\gamma^{2}-\alpha^{2}}-\gamma^{2}+\alpha^{2}}$ & center & - & - \\
\hline$y=o, x=\frac{\pi}{2}-\sqrt[3]{\frac{6 \beta}{\alpha}}$ & - & center & - \\
\hline$y=o, x=\pi-\arcsin \frac{\alpha}{\gamma}+\frac{\beta \alpha}{-\beta \sqrt{\gamma^{2}-\alpha^{2}}-\gamma^{2}+\alpha^{2}}$ & center & - & - \\
\hline
\end{tabular}

Investigation of the equations (1) by qualitative analyses and bifurcation methods permits to obtain the evolution traces at the various values of parameters for extra-solar planets. The obtained dynamical system may be naturally considered on the two-dimensional sphere.

Here we present the portrait, which are the most important in a view of their astronomical application. Possible equilibrium states and behavour of tracks in their vicinity are given in the Table 1 for positive values of parameters. 

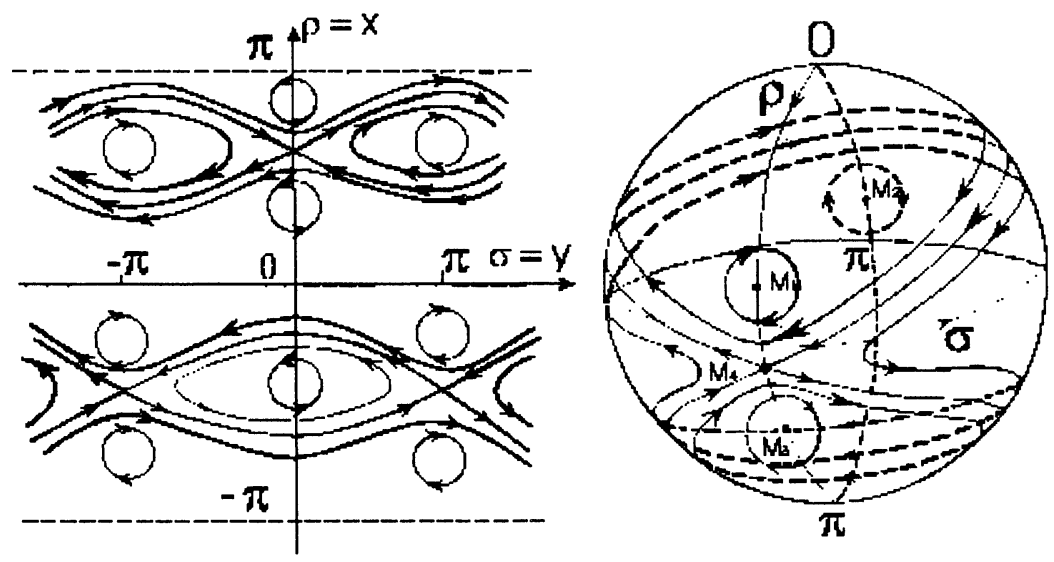

Figure 2. Phase portrait $\beta \neq 0$

Phase portrait, corresponding to the parameters values $\alpha<\gamma$ are shown on Fig. 2 on the phase plane and the sphere. Because, all variables in (1) are angles describing a position if the kinetic moment vector in a space, all phase portraits are convenient to consider on the unit 2D sphere $L=L_{0}$. The axis of ordinates shows the variation of the $\sigma$ angle along the latitude, and the axis of abscissa shows the variation of the $\rho$-angle along longitude.

Here are four stationary points $\mathrm{M}_{1}-\mathrm{M}_{4}$, three of them are of center kind $\left(\mathrm{M}_{1}, \mathrm{M}_{2}, \mathrm{M}_{3}\right)$ and one - of saddle kind $\mathrm{M}_{4}$. When the vector of kinetic moment falls in the equilibrium state (ES) vicinity $\mathrm{M}_{1}$, its motion will have type of precession with a constant angular distance $\rho^{*}, \sigma^{*}$ with periodical variation of the rotation from direct into reverse one. When moving away from the ES points, the oscillation period L will be increased. From the above, it may be concluded, that with certain values of parameters the motion of $\mathrm{L}$ may be changed from the direct kind into reverse one and vice versa.

\section{References}

Beletsky, V.V. \& Khentov, A.A. 1995, Resonance rotation of celestial mechanics, (N. Novg.), 430

Gonzalez, G. 1998, A\&A, 334, 221

Gusev, A. \& Kitiashvili, I. 1999, in Conf. Proc. Geom. of Physics IV, (Kazan: Kazan Univ. Press), 115

Kitiashvili, I.N. 1999, Evolution of the extrasolar planets. Master of Science Thesis, Kazan University, Kazan, 80

Mayor, M. \& Queloz, D. 1995, Nature, 378, 355

Nelson, A.F. \& Angel, J.R.P. 1998, ApJ, 500, 940

Wolszczan, A. \& Frail, D.A. 1992, Nature, 355, 145 